\title{
Optimal design for goodness-of-fit of the Michaelis-Menten enzyme kinetic function
}

\author{
Holger Dette \\ Ruhr-Universität Bochum \\ Fakultät für Mathematik \\ 44780 Bochum, Germany \\ e-mail: holger.dette@ruhr-uni-bochum.de
}

\author{
Viatcheslav B. Melas \\ St. Petersburg State University \\ Department of Mathematics \\ St. Petersburg, Russia \\ email: v.melas@pobox.spbu.ru
}

\author{
Weng Kee Wong \\ Dept. of Biostatistics \\ University of California \\ Los Angeles, CA 90095-1772, USA \\ email: wkwong@ucla.edu
}

\begin{abstract}
We construct efficient designs for the Michaelis-Menten enzyme kinetic model capable of checking model assumption. An extended model, called EMAX model is also considered for this purpose. This model is widely used in pharmacokinetics and reduces to the MichaelisMenten model for a specific choice of the parameter setting. Our strategy is to find efficient designs for estimating the parameters in the EMAX model and at the same time test the validity of the Michaelis-Menten model against the EMAX model by maximizing a minimum of the $D$ - or $D_{1}$-efficiencies taken over a range of values for the nonlinear parameters. In addition, we show that the designs obtained from maximizing the $D$-efficiencies are (i) efficient for estimating parameters in the EMAX model or the Michaelis-Menten model, (ii) efficient for testing the Michaelis-Menten model against the EMAX model and (iii) robust with respect to misspecification of the unknown parameters.
\end{abstract}

AMS Subject Classification: $62 \mathrm{~K} 05$

Keywords and Phrases: Chebyshev polynomials, EMAX model, goodness of fit test, locally Doptimal design, robust optimal design

\section{Introduction}

The motivation of our work here comes from the observation that the theoretical optimal designs for many models used in practice typically do not have enough design points to enable the re- 
searcher to verify the model assumptions. This is an important problem because optimal designs are model-dependent and can lose substantial efficiency under a mis-specified model. For example, the $D$-optimal design for a homoscedastic simple linear model is equally supported at the end points of the design interval but this design is completely useless for checking if a quadratic model provides a better fit. This is because the model has two parameters and the optimal design has only two distinct points. Consequently, there is no degree of freedom for lack of fit sum of squares and the test cannot be carried out. In other problems, the optimal design can have less support points than the number of parameters in the model and so does not even allow all parameters to be estimated.

There is very limited work to address this design issue to date especially for nonlinear models. In linear models, there has been some work in this direction. One way to overcome this problem is to embed the model in a class of plausible nested models constructed such that if the hypothesized model is not valid, anonther model in the class will provide an adequate fit. For instance, in simple linear regression problems with a continuous outcome, it is customary to model the data using a polynomial model of low degree first before attempting higher polynomial fits. Here, the class of models under consideration is the class of polynomials and each member is nested within polynomials of higher degrees. Using different design criteria, Stigler (1971), Studden (1982) and, Wong and Song (2002) constructed optimal designs for polynomial models when there is limited knowledge on the true degree of the model. Their design strategy was to embed the hypothesized model in a larger class of polynomial models. For instance, if the hypothesized model is quadratic, and polynomial models of degrees up to four are considered plausible, Studden (1982) provided an optimal design that would guarantee the smallest generalized variance for the estimated coefficients in the quadratic model subject to an additional requirement that the design also provides a userselected level of $D$-efficiency for estimating the coefficients associated with the cubic and quartic terms. The resulting design also has more than three support points and therefore a lack of fit test can be performed to assess if the quadratic model holds.

Work on related design issues for nonlinear models are virtually non-existent in the literature. One likely reason is that the design problem becomes very hard very quickly. This is not surprising because finding optimal design for a single nonlinear model can be a challenging task in itself. Lupinacci and Raghavarao (2000, 2002) appears among the first few to address similar design issues for nonlinear models. The first paper proposed adding an extra point for the D-optimal design for the simple logistic model to ensure symmetry in the variance function. The rationale was that with three design points and only two parameters in the model, the researcher can use the design to perform a lack of fit test. Using a similar reasoning, they proposed in the second paper that a third point be added mid-way between the two points obtained from the D-optimal design for the Michaelis-Menten model. In either case, we found their rationale was largely movtivated by convenience and light on statistical considerations. In the next few sections, we propose a technique for generating optimal designs capable of performing a lack of fit test for misspecification in nonlinear models. While the idea is a general one, specific details and technical difficulties will have to be dealt with for the specific models involved. To fix ideas, we will focus on a simple yet highly popular model in the applied sciences to demonstrate our technique.

The Michaelis Menten model is one of the most widely used models in the biological sciences. The model is perhaps most commonly used to study enzymatic reaction which is of great importance 
in pharmacology, biology and medical resarch. More specifically, the model is used to describe saturation functions for numerous physical and biological phenomena. Specific applications can be found in Cressie and Keightley (1979), Johansen (1984), Beverton and Holt (1957), CornishBrowden (1979), Hay, Meznarich, DiGiacomo, Hirst, Zerbe (1988), just to name a few. The simplest form encountered most often in biology is the familiar enzyme kinetic function

$$
y=\frac{a x}{b+x} ; \quad x \in\left[0, x_{0}\right]
$$

where $y$ is the reaction velocity, $a$ the maximum velocity of this reaction, $x$ the concentration of a substrate and $b$ the half-saturation constant, the value of $x$, where $y$ is half-maximal. Optimal designs for the Michaelis-Menten model have been studied by numerous authors [see e.g. Duggleby (1979), Dunn (1988), Rasch (1990), Boer, Rasch and Hendrix (2000), Dette and Wong (1999), Lopez-Fidalgo and Wong (2000) or Dette and Biedermann (2003) among many others].

In non-linear models, the Fisher information matrix depends on the unknown parameters and for this reason optimal designs, which maximize some function of the Fisher information matrix are difficult to implement in practice. Most authors concentrate on locally optimal designs, where it is assumed that a preliminary guess for the unknown parameters is available [see Chernoff (1953) or Silvey (1980)]. More recently, Song and Wong (1998) proposed Bayesian optimal designs and Dette and Biedermann (2003) suggested maximin $D$-optimal designs as robust alternatives for the Michaelis-Menten model. Both papers presented efficiencies of the optimal design when the nominal values are misspecified. See also Matthews and Allcock (2004) where they provided a variety of Bayesian optimal designs for the Michaelis-Menten model with applications in enzymology.

Most optimal designs for the Michaelis-Menten model have been criticized because they advise the experimenter to take observations only at two points. Consequently, these designs cannot be used to perform a goodness-of-fit test for the assumed model. We propose to embed the Michalis-Menten model in a larger class of models defined by

$$
E[Y \mid x]=\frac{a x^{h}}{b+x^{h}} ; \quad x \in\left[0, x_{0}\right]
$$

This model clearly contains the Michaelis-Menten model when $h=1$ and we will call it the EMAX model. This is the more common name in the literature, other names include the Sigmoid-EMAX model or the Hill model. The EMAX model is itself appealing in its own right and is a popular model in the biological sciences. For instance, in studies involving anticancer agents, the EMAX model is the conentration-effect curve with $x$ representing the concentration of the drug, $b$ the median effective concentration of the drug, $h$ is the curvature parameter (or the Hill's coefficient) and $y$ is the measured effect, such as percentage of cell surviving and $x$ is the concentraction of the drug. The EMAX model has many applications and is a serious competitor to the MichaelisMenten model [see Meftin, Winkle, Blaschke, Fitzgerald and Harrison (1977), Holford and Sheiner (1981) or Bezeau and Endrenyi (1986), for example]. Thus, estimating the Hill's coefficient has intrinsic value as well.

To test if the Michaelis-Menten model holds, we first assume the EMAX model holds and test the hypothesis

$$
H_{0}: h=1 \quad \text { vs } \quad H_{1}: h \neq 1 \text {. }
$$


It will be seen that optimal designs for testing the above hypothsis has more than two points and consequently, the researcher can carry out a formal lack of fit test.

Our first approach is based on the classical $D$-optimality criterion and maximizes the determinant of the (asymptotic) covariance matrix of the least squares estimator for the parameters $(a, b, h)$ in the model (1.2). The second method is based on the $D_{1}$-optimality criterion which determines the design such that the asymptotic variance of the least squares estimator for the parameter $h$ in the model (1.2) is minimal. Because the EMAX model is non-linear, the asymptotic covariance matrix depends on the parameters and this implies the optimal designs depend on the unknown parameters as well. However, it is easy to see that the optimal designs depend on $h$ and $b$ only and not on $a$. Following Chernoff (1953), we determine in Section 3 locally optimal designs, which require an initial guess of the unknown parameters. We show that these optimal designs are rather sensitive to the choice of the initial parameters. For this reason, we seek alternative strategies and construct in Section 4 standardized maximin optimal designs [Müller (1995) or Dette (1997), Imhof (2001) of Imhof and Wong (2000)] which maximize the minimum efficiency over a certain range of parameters. We show that these optimal designs are more robust to misspecification of the initial values than locally optimal designs. This is an important advantage because in many real experiments, initial values are usually vaguely known and a poor design can produce very inefficient estimates. In addition, the standardized maximin optimal designs are, on the one hand, quite efficient for discriminating between the Michaelis-Menten and the EMAX model and, on the other hand, also efficient for estimating the parameters in either the Michaelis-Menten model or the EMAX model. All justifications of our technical results are deferred to the appendix.

\section{Preliminaries}

Let $[0, T]$ denote the experimental region and assume that for each $x \in[0, T]$ an observation $Y$ could be made, where different observations are assumed to be independent with the same variance, say $\sigma^{2}>0$. Following Kiefer (1974) we call any probability measure

$$
\xi=\left(\begin{array}{ccc}
x_{1} & \ldots & x_{n} \\
w_{1} & \ldots & w_{n}
\end{array}\right)
$$

with finite support $x_{1}, \ldots, x_{n} \in[0, T], x_{i} \neq x_{j}(i \neq j), w_{i}>0, \sum_{i=1}^{n} w_{i}=1$ an experimental design. If $N$ is the total sample size, the experimenter takes approximately $n_{i} \approx N w_{i}$ observations at $x_{i}(i=1, \ldots, n)$ such that $\sum_{i=1}^{n} n_{i}=N$. It is well known [see Jennrich (1969)] that under regularity assumptions, the asymptotic covariance matrix of the least squares estimator $\hat{\theta}=(\hat{a}, \hat{b}, \hat{h})^{T}$ for the parameter $\theta=(a, b, h)^{T}$ in the model (1.2) is given by the matrix $\frac{\sigma^{2}}{N} M^{-1}(\xi, a, b, h)$. Here we assume the design $\xi$ has at least three points,

$$
M(\xi, a, b, h)=\int_{0}^{T} f(x, a, b, h) f^{T}(x, a, b, h) d \xi(x)
$$

is the information matrix of the design $\xi$ for the EMAX model (1.2) and

$$
f(x, a, b, h)=\frac{x^{h}}{b+x^{h}}\left(1,-\frac{a}{b+x^{h}}, \frac{a b \log x}{b+x^{h}}\right)^{T}
$$


is the vector of partial derivatives of the conditional expectation $E[Y \mid x]=a x^{h} /\left(b+x^{h}\right)$ with respect to the parameters $a, b$ and $h$. For a fixed $\theta=(a, b, h)^{T}$ a locally $D$-optimal design $\xi_{a, b, h}^{D}$ maximizes the determinant $|M(\xi, a, b, h)|$, while the locally $D_{1}$-optimal design $\xi_{a, b, h}^{D_{1}}$ maximizes

$$
\left(e_{3}^{T} M^{-1}(\xi, a, b, h) e_{3}\right)^{-1}=\frac{|M(\xi, a, b, h)|}{|\tilde{M}(\xi, a, b, h)|},
$$

where $e_{3}^{T}=(0,0,1)$ and the matrix $\tilde{M}$ is obtained from $M$ by deleting the last row and column. The locally $D$-optimal design minimizes the first order approximation of volume of the ellipsoid of concentration for the parameter $\theta$ [see e.g. Silvey (1980)], while the local $D_{1}$-optimal design minimizes the asymptotic variance of the least squares estimate for the unknown parameter $h$ and therefore maximizes the power of the test for the hypothesis (1.3). As a consequence, designs maximizing (2.4) are very efficient for discriminating between the Michaelis-Menten and EMAX model.

It is easy to see that the locally $D_{1}$-and $D$-optimal design do not depend on the parameter $a$ and for this reason we will set $a=1$ throughout this paper. Because we are interested in designs which are efficient for discriminating between the Michaelis-Menten and the EMAX model in (1.2) we put $h=1$ throughout this paper. This choice is also partially motivated by the following result which describes the relationship among the locally optimal designs for different values of $h$.

Theorem 2.1. Let $\xi$ denote a locally D-optimal (D1-optimal) design for the EMAX model (1.2) on the interval $[0, T]$ with parameters $(b, h)$ and define $\tilde{\xi}$ as the design on the interval $\left[0, T^{H}\right]$, which is obtained from the design $\xi_{a, b, h}$ by the transformation $x \rightarrow x^{h}$, then $\tilde{\xi}$ is D-optimal (D-optimal) on the interval $\left[0, T^{h}\right]$ for the model (1.2) with parameters $(b, 1)$.

Note that Theorem 2.1 allows to relate locally $D$-optimal designs for the EMAX model on different design spaces. Consider for example a locally $D$-optimal design on the interval $[0, T]$ when $\theta=$ $(a, b, 1)^{T}$ with support points $x_{i}$ and weights $w_{i}$. Then the design which puts masses $w_{i}$ at the points $x_{i}^{h}$ is locally $D$-optimal when $\theta=(a, b, h)^{T}$ for the EMAX model on the interval $\left[0, T^{h}\right]$. In other words, if the locally $D$-optimal designs for $h=1$ and any $b$ and $T$ are known, the locally optimal designs for any parameter $\theta=(a, b, h)^{T}$ and any design space can easily be derived. A further reduction of the locally optimal design problem will be obtained in the following section. In what is to follow, we denote for simplicity $M(\xi, b)=M(\xi, 1, b, 1), f(x, b)=f(x, 1, b, 1)$ and define $\xi_{b}^{D}$ and $\xi_{b}^{D_{1}}$ as the locally $D$ and $D_{1}$-optimal design at the point $\theta=(1, b, 1)^{T}$, respectively.

\section{Locally optimal designs}

Due to the complexity of function $f(x, a, b, h)$ it is not possible to construct the locally optimal designs for the EMAX model explicitly. Bezeau and Endrenyi (1986) found locally D-optimal designs numerically for the EMAX model. In this section we demonstrate that it is possible to establish general properties of locally $D$ - and $D_{1}$-optimal designs and use them to simplify our calculation of the locally optimal designs in a substantial way. We begin with some important properties of the $D$-optimal design. 
Theorem 3.1. The locally D-optimal design $\xi_{b}^{D}$ in the EMAX model (1.2) on the interval $[0,1]$ is unique and has equal masses at three points including the boundary point $x_{3}=1$.

Moreover, the locally D-optimal design in the EMAX model (1.2) on the interval [0,T] with parameters $(b, 1))$ is obtained from the locally D-optimal design $\xi_{b / T}^{D}$ on the interval $[0,1]$ with parameters $(b / T, 1))$ by multiplying the corresponding support points by $T$.

Theorems 2.1 and 3.1 simplify the determination of locally $D$-optimal designs for the EMAX model substantially. If a locally $D$-optimal design on the interval $[0, T]$ with parameters $(b, h)$ is sought, we determine the locally $D$-optimal design $\xi_{b / T^{h}}^{D}$ on the interval $[0,1]$ for the parameters $\left(b / T^{h}, 1\right)$. By Theorem 3.1 the design $\xi_{b}^{D}$ defined by the transformation

$$
\xi_{b}^{D}(\{x\})=\xi_{b / T^{h}}^{D}\left(\left\{\frac{x}{T^{h}}\right\}\right)
$$

is locally $D$-optimal for the EMAX model on the interval $\left[0, T^{h}\right]$ for the parameters $(b, 1)$ and Theorem 2.1 shows that the design $\xi_{b, h}^{D}$ obtained by the transformation

$$
\xi_{b, h}^{D}(\{x\})=\xi_{b}^{D}\left(\left\{x^{h}\right\}\right)=\xi_{b / T^{h}}^{D}\left(\left(\frac{x}{T}\right)^{h}\right)
$$

is locally $D$-optimal for the EMAX model on the interval $[0, T]$ for the parameters $(b, h)$. Therefore it is sufficient to calculate locally $D$-optimal designs on the interval $[0,1]$ for the parameters $(b, 1)$. The first part of Theorem 3.1 shows that we can restrict ourselves to designs of the form

$$
\xi_{b}^{D}=\left(\begin{array}{ccc}
x_{1} & x_{2} & 1 \\
\frac{1}{3} & \frac{1}{3} & \frac{1}{3}
\end{array}\right) ; 0<x_{1}<x_{2}<1
$$

For this type of design the determinant of the information matrix in the EMAX model reduces to $\left|M\left(\xi_{b}^{D}, b\right)\right|=c \Phi^{2}\left(x_{1}, x_{2}, b\right)$, where the constant $c$ does not depend on $x_{1}, x_{2}$ and the function $\Phi$ is defined by

$$
\Phi\left(x_{1}, x_{2}, b\right)=\frac{b\left[\left(x_{2}-1\right) \log x_{1}-\left(x_{1}-1\right) \log x_{2}\right]}{27(1+b)^{2}\left(b+x_{1}\right)^{2}\left(b+x_{2}\right)^{2}} .
$$

This function can easily be maximized numerically. In Table 3.1 we present some locally $D$-optimal designs obtained by the Nelder-Mead method. The optimality of the designs over the set of all designs defined on $[0,1]$ was verified using an equivalence theorem which is widely discussed in design monographs, see Silvey (1980) for example. We observe from the table that the locally $D$-optimal designs depend sensitively on the choice of the parameter $b$.

In order to illustrate the application of Theorem 2.1 and 3.1 consider the locally $D$-optimal design problem for the EMAX model on the interval $[0,2]$ when $h=2, b=2$. The locally $D$-optimal design on the interval $[0,1]$ for the parameters $\tilde{h}=1$ and $\tilde{b}=b / T^{h}=0.05$ is obtained from Table 3.1 and has equal masses at the points $0.073,0.388$ and 1 . From equation (3.1) it follows that the locally $D$-optimal design problem for the EMAX model on the interval [0,2] with $h=2$, $b=2$ advices the experimenter to take the same proportion of total observations at the points $2 \cdot 0.073^{0.5}=0.540,2 \cdot 0.388^{0.5}=1.246$ and 2 . 


\begin{tabular}{|c|cc||c|cc|}
\hline$b$ & $x_{1}$ & $x_{2}$ & $b$ & $x_{1}$ & $x_{2}$ \\
\hline 0.1 & 0.026 & 0.171 & 1.1 & 0.100 & 0.482 \\
0.2 & 0.042 & 0.260 & 1.2 & 0.103 & 0.491 \\
0.3 & 0.055 & 0.317 & 1.3 & 0.106 & 0.498 \\
0.4 & 0.065 & 0.358 & 1.4 & 0.108 & 0.505 \\
0.5 & 0.073 & 0.388 & 1.5 & 0.110 & 0.511 \\
0.6 & 0.079 & 0.412 & 1.6 & 0.112 & 0.516 \\
0.7 & 0.085 & 0.431 & 1.7 & 0.114 & 0.521 \\
0.8 & 0.089 & 0.447 & 1.8 & 0.116 & 0.525 \\
0.9 & 0.094 & 0.461 & 1.9 & 0.117 & 0.529 \\
1.0 & 0.097 & 0.473 & 2.0 & 0.118 & 0.533 \\
\hline
\end{tabular}

Table 3.1. Interior support points $\left(x_{1}, x_{2}\right)$ of the equally weighted locally D-optimal designs $\xi^{D}$ for the EMAX model (1.2) on the interval [0,1] with $h=1$ and different values of $b$. The equally weighted locally D-optimal designs for the EMAX model (1.2) on the interval [0,T] are obtained by the transformation (3.1).

The locally $D_{1}$-optimal designs can be obtained by similar methods. More precisely, we show in the appendix that the functions

$$
\frac{x}{(x+b)^{2}}, \frac{x^{2}}{(x+b)^{2}}, \frac{x \log x}{(x+b)^{2}}
$$

generate a Chebyshev system on the interval $(0, T]$ [see Karlin and Studden (1966)]. As a consequence, there exists constants $\alpha_{1}, \alpha_{2}, \alpha_{3} \in \mathbb{R}$ such that the function

$$
\psi(x)=(x+b)^{-2}\left\{\alpha_{1} x+\alpha_{2} x^{2}+\alpha_{3} x \log x\right\}
$$

oscillates on the interval $[0,1]$ at three points, say $0<x_{1}^{*}<x_{2}^{*}<x_{3}^{*}=T$, which means

$$
\begin{aligned}
& |\psi(x)| \leq 1 \quad \text { for all } x \in[0,1] \\
& \left|\psi\left(x_{i}^{*}\right)\right|=(-1)^{i} \quad i=1,2,3 .
\end{aligned}
$$

These points are uniquely determined and are called Chebyshev points in the literature. The corresponding function $\psi$ is called a Chebyshev polynomial. The Chebyshev points depend on the values of $b$ and $T$ and it is sometimes helpful to make this dependence explicit by writing $x_{i}^{*}$ as $x_{i}^{*}(b, T)$. It is easy to see that

$$
x_{i}^{*}(b, T)=T x_{i}^{*}\left(\frac{b}{T}, 1\right) \quad i=1,2,3
$$

and consequently it is sufficient to present these points for the interval $[0,1]$ for which the corresponding Chebyshev points are denoted by $x_{i}^{*}(b)[i=1,2,3]$. We are now in a position to state an analogue of Theorem 3.1. 
Theorem 3.2. For $b>0$ let $x_{1}^{*}(b), x_{2}^{*}(b), x_{3}^{*}(b)=1$ denote the points determined by (3.6). The locally $D_{1}$-optimal design $\xi_{b}^{D_{1}}$ for the EMAX model (1.2) with parameters $\left.(b, 1)\right)$ on the interval $[0, T]$ is uniquely given by

$$
\xi_{D_{1}}^{*}=\left(\begin{array}{ccc}
T x_{1}^{*}\left(\frac{b}{T}\right) & T x_{2}^{*}\left(\frac{b}{T}\right) & T \\
w_{1}^{*}\left(\frac{b}{T}\right) & w_{2}^{*}\left(\frac{b}{T}\right) & w_{3}^{*}\left(\frac{b}{T}\right)
\end{array}\right)
$$

where the weights $w_{1}, w_{2}, w_{3}$ are given by

$$
w_{i}^{*}(\beta)=\frac{A_{i}(\beta)}{A_{1}(\beta)+A_{2}(\beta)+A_{3}(\beta)} \quad i=1,2,3
$$

with

$$
\begin{aligned}
& A_{1}(\beta)=x_{a}^{*}(\beta)\left(1-x_{1}^{*}(\beta)\right)\left(\beta+x_{2}^{*}(\beta)\right)^{2} \\
& A_{2}(\beta)=x_{1}^{*}(\beta)\left(1-x_{1}^{*}(\beta)\right)\left(\beta+x_{1}^{*}(\beta)\right)^{2} \\
& A_{3}(\beta)=x_{1}^{*}(\beta) x_{2}^{*}(\beta)(\beta+1)^{2}
\end{aligned}
$$

It follows from Theorem 3.2 that it is sufficient to consider the case $T=1$, for which the $D_{1}$-optimal design is of the form

$$
\xi=\left(\begin{array}{ccc}
x_{1} & x_{2} & 1 \\
w_{1} & w_{2} & 1-w_{1}-w_{2}
\end{array}\right)
$$

Moreover, we have from the proof of Theorem 3.2 in the appendix that

$$
\min _{w_{1}, w_{2} \in(0,1)} e_{3}^{T} M^{-1}(\xi, b) e_{3}=\rho^{2}\left(x_{1}, x_{2}, b\right),
$$

where the function $\rho$ is defined by

$$
\rho\left(x_{1}, x_{2}, b\right)=-\frac{x_{2}\left(1-x_{2}\right)\left(b+x_{1}\right)^{2}+x_{1}\left(1-x_{1}\right)\left(b+x_{2}\right)^{2}+x_{1} x_{2}\left(x_{2}-x_{1}\right)(1+b)^{2}}{x_{1} x_{2}\left[\left(1-x_{2}\right) \log x_{1}-\left(1-x_{1}\right) \log x_{2}\right]}
$$

It follows that for a given value of $b$, the support points of the locally $D_{1}$-optimal design can easily be found numerically by minimizing the function $\rho^{2}$ with respect to $0 \leq x_{1}<x_{2} \leq 1$. In Table 3.2 , we show numerical locally $D_{1}$-optimal designs for the EMAX model (1.2), where $h=1$ and selected values of $b$ in the interval $[0.1,2]$. We observe again that the locally $D_{1}$-optimal design EMAX model (1.2) depends sensitively on the value of the parameter $b$. 


\begin{tabular}{|c|cc|ccc|}
\hline$b$ & $x_{1}$ & $x_{2}$ & $w_{1}$ & $w_{2}$ & $w_{3}$ \\
\hline & & & & & \\
0.1 & 0.014 & 0.179 & 0.553 & 0.305 & 0.142 \\
0.2 & 0.023 & 0.270 & 0.576 & 0.293 & 0.130 \\
0.3 & 0.030 & 0.328 & 0.591 & 0.286 & 0.123 \\
0.4 & 0.036 & 0.370 & 0.601 & 0.280 & 0.119 \\
0.5 & 0.041 & 0.401 & 0.609 & 0.276 & 0.115 \\
0.6 & 0.045 & 0.425 & 0.615 & 0.273 & 0.112 \\
0.7 & 0.048 & 0.444 & 0.620 & 0.270 & 0.110 \\
0.8 & 0.051 & 0.460 & 0.624 & 0.268 & 0.108 \\
0.9 & 0.054 & 0.474 & 0.627 & 0.266 & 0.107 \\
1 & 0.056 & 0.485 & 0.630 & 0.264 & 0.106 \\
1.1 & 0.058 & 0.495 & 0.633 & 0.263 & 0.105 \\
1.2 & 0.060 & 0.503 & 0.635 & 0.262 & 0.104 \\
1.3 & 0.062 & 0.511 & 0.637 & 0.260 & 0.103 \\
1.4 & 0.063 & 0.517 & 0.639 & 0.259 & 0.102 \\
1.5 & 0.064 & 0.523 & 0.640 & 0.258 & 0.101 \\
1.6 & 0.066 & 0.529 & 0.642 & 0.258 & 0.101 \\
1.7 & 0.067 & 0.533 & 0.643 & 0.257 & 0.100 \\
1.8 & 0.068 & 0.538 & 0.644 & 0.256 & 0.100 \\
1.9 & 0.069 & 0.542 & 0.645 & 0.256 & 0.099 \\
2 & 0.070 & 0.545 & 0.646 & 0.255 & 0.099 \\
\hline
\end{tabular}

Table 3.2. Locally $D_{1}$-optimal designs for the EMAX model (1.2) on the interval [0,1] with $h=1$ and different values of the parameter $b$. The third support is given by $x_{3}=1$.

\section{Robust and efficient designs}

The numerical results of the previous sections show that the locally $D$ - and $D_{1}$-optimal designs are not necessarily robust with respect to the choice of the initial parameters. In order to obtain designs that are efficient and robust over a certain range of the parameters for the EMAX model, we study a maximin approach proposed by Müller (1995) and Dette (1997). To be precise we define

$$
I_{D}(\xi)=\min _{b \in[b, \bar{b}]} \frac{\mid M(\xi, b \mid)}{\max _{\eta}|M(\eta, b)|}
$$

as the minimal efficiency of the design $\xi$ if $b$ varies in the interval $[\underline{b}, \bar{b}]$, and call a design $\xi^{D}$ standardized maximin $D$-optimal for the EMAX model, if it maximizes the optimality criterion in (4.1). Similarly, a design $\xi^{D_{1}}$ is called standardized maximin $D_{1}$-optimal, if it maximizes

$$
I_{D_{1}}(\xi)=\min _{b \in[\underline{b}, \bar{b}]} \frac{\left(e_{3}^{T} M^{-1}(\xi, b) e_{3}\right)^{-1}}{\max _{\eta}\left(e_{3}^{T} M^{-1}(\eta, b) e_{3}\right)^{-1}} .
$$


Our first result shows that when we calculate the standardized maximin optimal designs, it is again sufficient to consider the interval $[0,1]$ as our design space. The justification is given in the appendix.

Lemma 4.1. If $\xi$ is the standardized maximin $D-\left(D_{1}\right)$-optimal design for the EMAX model on the interval $[0,1]$ with respect to the interval $[\underline{b}, \bar{b}]$, then the design $\xi_{T}$ induced by the transformation $\xi_{T}(\{x\})=\xi\left(\left\{\frac{x}{T}\right\}\right)$ is standardized maximin $D-\left(D_{1}\right)$-optimal on the interval $[0, T]$ with respect to the interval $[\underline{b} / T, \bar{b} / T]$.

In general the determination of standardized maximin optimal designs has to be done numerically. In our study we determined the optimal designs on the interval $[0,1]$ within the class of all designs with three support points numerically and verified the optimality within the class of all designs by an application of the following equivalence theorem [see Dette, Haines and Imhof (2003)].

\section{Theorem 4.2.}

(a) A design is $\xi^{D}$ standardized maximin D-optimal if and only if there exists a prior distribution $\pi$ on the set

$$
\left\{b \in[\underline{b}, \bar{b}] \mid I_{D}\left(\xi^{D}\right)=\frac{\left|M\left(\xi^{D}, b\right)\right|}{\max _{\eta}|M(\eta, b)|}\right\}
$$

such that the inequality

$$
\int_{\underline{b}}^{\bar{b}} f(t, b) M^{-1}\left(\xi^{D}, b\right) f(t, b) \pi(d b) \leq 3
$$

holds for all $t \in[0, T]$. Moreover, there is equality in (4.3) for all support points of the standardized maximin D-optimal design $\xi^{D}$.

(b) $A$ design $\xi^{D}$ is standardized maximin $D_{1}$-optimal if and only if there exists a prior on the set

$$
\left\{b \in[\underline{b}, \bar{b}] \mid I_{D_{1}}(\xi)=\frac{\left(e_{3}^{T} M^{-1}\left(\xi^{D}, b\right) e_{3}\right)^{-1}}{\sup _{\eta}\left(e_{3}^{T} M^{-1}(\eta, b) e_{3}\right)^{-1}}\right\}
$$

such that the inequality

$$
\int_{\underline{b}}^{\bar{b}}\left(f^{T}(t, b) M^{-1}\left(\xi^{D_{1}}, b\right) e_{3}\right)^{2} \pi(d b) \leq I_{D_{1}}\left(\xi^{*}\right)
$$

holds for all $t \in[0, T]$. Moreover, there is equality in (4.4) for all support points of the standardized maximin $D_{1}$-optimal design $\xi^{D_{1}}$.

In Tables 4.1 and 4.2 we display selected numerical standardized maximin $D$ - and $D_{1}$-optimal designs on different intervals $[\underline{b}, \bar{b}]$. Again we restrict ourselves to the case $T=1$ [see Lemma 4.1] and $h=1$, which is most important for the discrimination between the EMAX and MichaelisMenten model. In all cases we started with the calculation of standardized maximin optimal designs in the class of all three point designs. The optimality was checked by an application of Theorem 4.2. If the optimal three point design was not optimal within the class of all designs a 
fourth support point was added and the calculation was repeated in order to obtain a better design with four support points. In all cases considered in our study the standardized maximin optimal designs have at most four support points. For example, only in the cases $\underline{b}=0.1, \bar{b} \in\{1,2\}$ the standardized maximin $D$-optimal design is supported on four points including the right endpoint, while in all other cases a three point design is sufficient [see Table 4.1].

\begin{tabular}{|c|c|c|c|c|c|c|c|c|c|c|}
\hline$\underline{b}$ & $\bar{b}$ & $x_{0}$ & $x_{1}$ & $x_{2}$ & $x_{3}$ & $\omega_{0}$ & $\omega_{1}$ & $\omega_{2}$ & $\omega_{3}$ & $I_{D}(\xi)$ \\
\hline 0.1 & 2 & .0325 & .1496 & .5111 & 1 & .2100 & .2604 & .2250 & .3046 & .875 \\
0.1 & 1 & .0330 & .1394 & .4427 & 1 & .2291 & .2312 & .2277 & .3120 & .885 \\
0.3 & 1 & & .0722 & .3945 & 1 & & $1 / 3$ & $1 / 3$ & $1 / 3$ & .969 \\
0.3 & 2 & & 0.789 & .4260 & 1 & & $1 / 3$ & $1 / 3$ & $1 / 3$ & .944 \\
1 & 2 & & .1071 & .5030 & 1 & & $1 / 3$ & $1 / 3$ & $1 / 3$ & .995 \\
1 & 5 & & .1149 & .5272 & 1 & & $1 / 3$ & $1 / 3$ & $1 / 3$ & .987 \\
1 & 10 & & .1179 & .5367 & 1 & & $1 / 3$ & $1 / 3$ & $1 / 3$ & .982 \\
1 & 20 & & .1195 & .5418 & 1 & & $1 / 3$ & $1 / 3$ & $1 / 3$ & .979 \\
1 & 50 & & .1205 & .5449 & 1 & & $1 / 3$ & $1 / 3$ & $1 / 3$ & .978 \\
\hline
\end{tabular}

Table 4.1. Standardized maximin D-optimal designs for the EMAX model (1.2) on the interval $[0,1]$ with respect to the interval $[\underline{b}, \bar{b}]$. The table shows the support points, weights and the minimal efficiency of the standardized maximin D-optimal design over the range $[\underline{b}, \bar{b}]$.

\begin{tabular}{|c|c|c|c|c|c|c|c|c|c|c|}
\hline$\underline{b}$ & $\bar{b}$ & $x_{0}$ & $x_{1}$ & $x_{2}$ & $x_{3}$ & $\omega_{0}$ & $\omega_{1}$ & $\omega_{2}$ & $\omega_{3}$ & $I_{D}(\xi)$ \\
\hline 0.1 & 2 & .0239 & .1319 & .4881 & 1 & .4733 & .2419 & .1758 & .1090 & .658 \\
0.1 & 1 & .0214 & .1188 & .4487 & 1 & .4754 & .2319 & .1816 & .1111 & .669 \\
0.3 & 1 & & .0426 & .3908 & 1 & & .6069 & .2721 & .1209 & .868 \\
0.3 & 2 & & 0.483 & .4091 & 1 & & .6121 & .2659 & .1220 & .779 \\
1 & 2 & & .0627 & .5128 & 1 & & .6378 & .2591 & .1031 & .981 \\
1 & 5 & & .0684 & .5314 & 1 & & .6436 & .2540 & .1024 & .942 \\
1 & 10 & & .0708 & .5380 & 1 & & .6450 & .2527 & .1023 & .923 \\
1 & 20 & &. .0721 & .5413 & 1 & & .6461 & .2517 & .1022 & .912 \\
1 & 50 & & .0729 & .5434 & 1 & & .6467 & .2510 & .1023 & .905 \\
\hline
\end{tabular}

Table 4.2. Standardized maximin $D_{1}$-optimal designs for the EMAX model (1.2) on the interval $[0,1]$ with respect to the interval $[\underline{b}, \bar{b}]$. The table shows the support points, weights and the minimal efficiency of the standardized maximin $D_{1}$-optimal design over the range $[\underline{b}, \bar{b}]$.

We observe that this property depends on the size of the ratio $\underline{b} / \bar{b}$ [see also Dette and Biedermann (2003) who made a similar observation for the Michaelis-Menten model]. Moreover, the standardized maximin $D$-optimal design yields rather high $D$-efficiencies for a broad range of $b$ values. For 
example, if $b$ varies in the interval $[1,50]$ the minimal efficiency of the standardized maximin $D$ optimal design is $97.8 \%$ and even in the case where the ratio $\bar{b} / \underline{b}$ is 200 the standardized maximin $D$-optimal design has at least efficiency $87.5 \%$.

The situation for the standardized $D_{1}$-optimal design is very similar and illustrated in Table 4.2. Only in the case $\bar{b} / \underline{b} \geq 100$ four point designs are required for the standardized maximin $D_{1^{-}}$ optimal design and for $\bar{b} / \underline{b} \leq 50$ the minimal $D_{1}$-efficiency of the standardized maximin $D_{1}$-optimal design calculated over the interval $[\underline{b}, \bar{b}]$ is at least $90.5 \%$.

\begin{tabular}{|c|c|c|c|c|c|c|c|c|c|c|}
\hline \multicolumn{2}{|c|}{} & \multicolumn{3}{|c|}{$b=\underline{b}$} & \multicolumn{3}{c|}{$b=\bar{b}$} & \multicolumn{3}{c|}{$b=\frac{1}{2}(\underline{b}+\bar{b})$} \\
\hline$\underline{b}$ & $\underline{b}$ & $\kappa_{1}$ & $\kappa_{2}$ & $\kappa_{3}$ & $\kappa_{1}$ & $\kappa_{2}$ & $\kappa_{3}$ & $\kappa_{1}$ & $\kappa_{2}$ & $\kappa_{3}$ \\
\hline 1 & 2 & 1.18 & 1.07 & 0.68 & 0.99 & 0.94 & 0.67 & 1.05 & 0.98 & 0.67 \\
1 & 5 & 1.22 & 1.11 & 0.69 & 0.91 & 0.90 & 0.67 & 0.96 & 0.93 & 0.67 \\
1 & 10 & 1.23 & 1.12 & 0.69 & 0.89 & 0.88 & 0.67 & 0.92 & 0.91 & 0.67 \\
1 & 20 & 1.23 & 1.13 & 0.69 & 0.88 & 0.88 & 0.67 & 0.89 & 0.89 & 0.67 \\
1 & 50 & 1.24 & 1.13 & 0.69 & 0.87 & 0.87 & 0.67 & 0.88 & 0.88 & 0.67 \\
.5 & 1 & 1.29 & 1.09 & 0.70 & 1.02 & 0.94 & 0.69 & 1.12 & 0.99 & 0.69 \\
.3 & 1 & 1.41 & 1.12 & 0.72 & 0.97 & 0.91 & 0.73 & 1.09 & 0.96 & 0.71 \\
\hline
\end{tabular}

Table 4.3. Efficiencies of the $D_{1}$-optimal standardized maximin optimal design for estimating the individual coefficients in the EMAX model (1.2). The efficiency is calculated with respect to the standardized maximin D-optimal design and defined in (4.5).

We also compare the performance of the standardized maximin $D$ - and $D_{1}$-optimal design $\xi^{D}$ and $\xi^{D_{1}}$ by calculating the efficiency of the design $\xi^{D_{1}}$ with respect to the design $\xi^{D}$ for estimating the individual coefficients $a, b, h$. Specifically, we compute the ratio

$$
\kappa_{i}(b)=\frac{e_{i}^{T} M^{-1}\left(\xi^{D_{1}}, b\right) e_{i}}{e_{i}^{T} M^{-1}\left(\xi^{D}, b\right) e_{i}} \quad i=1,2,3,
$$

where $e_{i}$ denotes the $i$ th unit vector in $\mathbb{R}^{3}(i=1,2,3)$. The results are listed in Table 4.3. Note a value smaller than 1 for $\kappa_{i}(b)$ indicates that for the specific value of $b$ the standardized maximin $D_{1}$-optimal design $\xi^{D_{1}}$ is more efficient for estimating the corresponding parameter than the standardized maximin $D$-optimal design $\xi^{D}$, where $i=1,2,3$ correspond to the parameters $a, b$ and $h$, respectively. As expected the standardized maximin $D_{1}$-optimal design is substantially more efficient for estimating the parameter $h$. On the other hand the situation for the parameters $a$ and $b$ is not so clear any more. Here we observe that for the lower boundary $b=\underline{b}$ the standardized maximin $D$-optimal design $\xi^{D}$ yields smaller variances for least squares estimates of the parameters $a$ and $b$. This improvement can be substantial. For example, if $\underline{b}=0.3$ and $\bar{b}=1$, the standardized maximin $D$-optimal design is $40 \%$ more efficient for estimating the parameter $a$ in the EMAX model if $b=0.3$ would be the "true" parameter. This superiority decreases if $b$ moves to right boundary $\bar{b}$. Here the efficiencies vary between $87 \%$ and $102 \%$, but the standardized maximin $D$-optimal design still yields reasonable efficiencies. 
These results suggest that the standardized maximin $D$-optimal designs for the EMAX model are more efficient for inference in the Michaelis-Menten model, if the null-hypothesis in (1.3) cannot be rejected. In order to study the loss of efficiency if the standardized maximin optimal designs from the EMAX model are used in the Michaelis-Menten model (1.1) we investigate the $D$-efficiency

$$
\operatorname{eff}_{D}(\xi, b)=\left(\frac{|\bar{M}(\xi, b)|}{\sup _{\eta}|\bar{M}(\eta, b)|}\right)^{1 / 2}
$$

where

$$
\bar{M}(\xi, b)=\int_{0}^{T} \frac{x^{2}}{(b+x)^{2}}\left(\begin{array}{cc}
1 & -\frac{a}{b+x} \\
-\frac{a}{b+x} & \frac{a^{2}}{(b+x)^{2}}
\end{array}\right) d \xi(x)
$$

is the information matrix of the design $\xi$ in the Michaelis-Menten model. Note that this efficiency does not depend on the parameter $a$. In Figure 4.1 we present these efficiencies for the standardized maximin $D$ - and $D_{1}$-optimal design for the EMAX model and for the standardized maximin $D$ optimal design for the Michaelis-Menten model, which was recently determined by Dette and Biedermann (2003). We considered the cases, where the interval for the parameter $b$ is given by $[0.3,1],[0.3,2],[1,5]$ and $[1,10]$ and the picture is very similar for all situations. The standardized maximin $D_{1}$-optimal design for the EMAX model yields only efficiencies about $40 \%$ for the estimation of the parameters in the Michaelis-Menten model and cannot be recommended if the experimenter is mainly interested in a design for the Michaelis-Menten model which allows the possibility of checking the goodness-of-fit of the EMAX model. The standardized maximin $D$ optimal designs for the Michaelis-Menten model are two point designs, robust and very efficient for inference in the Michaelis-Menten model. However, these designs cannot be used for testing the hypothesis (1.3). On the other hand the standardized maximin $D$-optimal designs from the EMAX model have at least three support points and therefore allow to check the model assumptions of the Michaelis-Menten enzyme kinetic function. If the hypothesis (1.3) of the EMAX model cannot be rejected these designs have about $75 \% D$-efficiency for inference in the Michaelis-Menten model. For this reason these designs are recommended for inference with the Michaelis-Menten enzyme kinetic function, because they are quite robust with respect to a misspecification of the range for the nonlinear parameter $b$ and additionally allow to check the model assumptions by testing the hypothesis (1.3).

Acknowledgements: The authors would like to thank A. Pepelyshev for computational assistance and Isolde Gottschlich, who typed most parts of this paper with considerable technical expertise. Parts of this paper were written during a visit of the first author at Purdue University and this author would like to thank the Department of Statistics for their hospitality. The support of the Deutsche Forschungsgemeinschaft (DE 502/14-1, 436 RUS 113/712/0-1 and SFB 475, Komplexitätsreduktion in multivariaten Datenstrukturen, Teilprojekt A2) is gratefully acknowledged. 

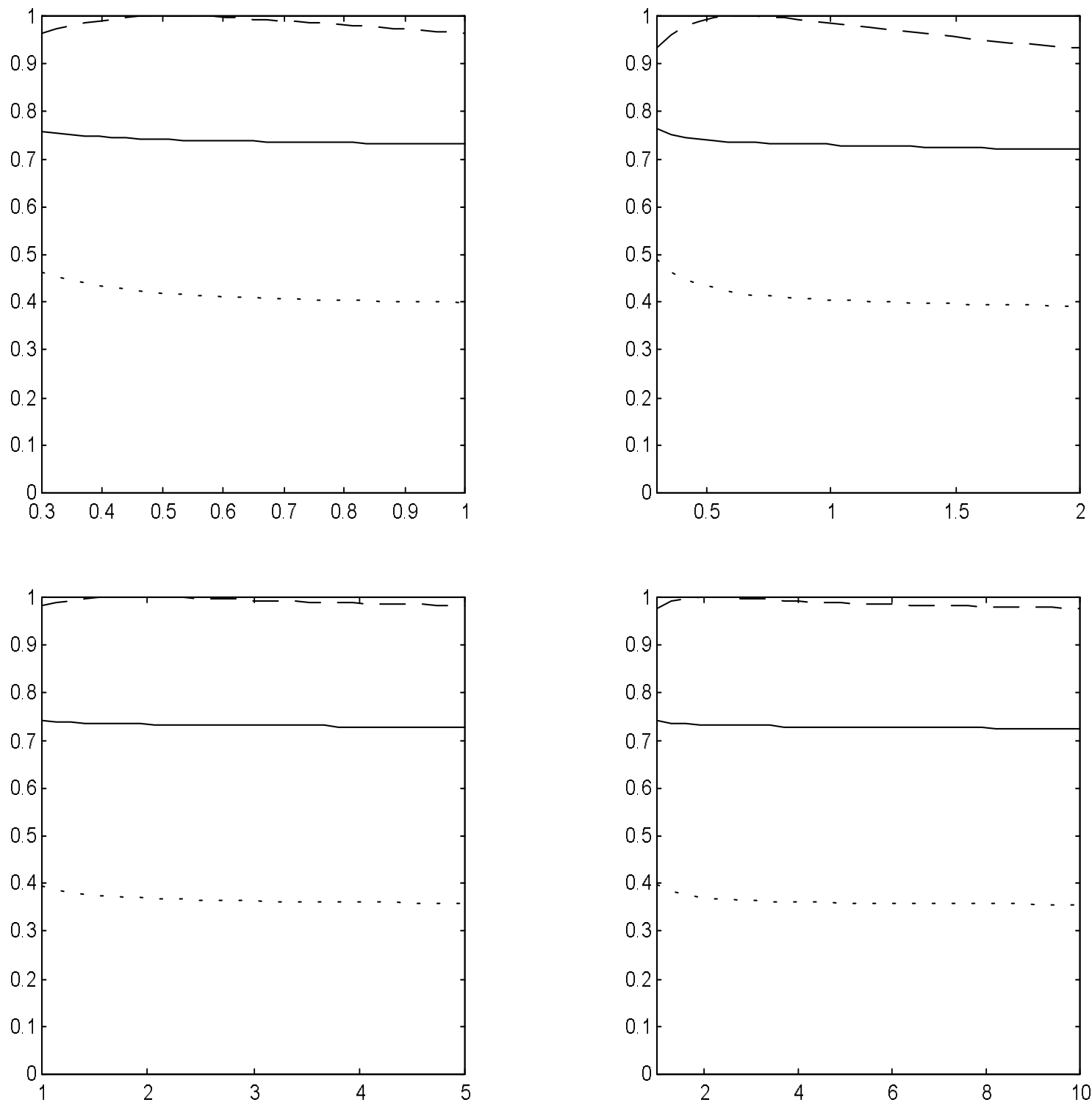

Figure 1: D-efficiencies (4.6) in the Michaelis-Menten model (1.1) of the standardized maximin D-optimal (solid line) and standardized maximin D -optimal design (dotted line) for the EMAX model (1.2). The figures also contain the efficiencies of the standardized maximin D-optimal designs for the Michaelis-Menten model (dashed line). The range for the parameter $b$ in the standardized maximin criteria is given by $[\underline{b}, \bar{b}]=[0.3,1]$ (left upper panel), $[\underline{b}, \bar{b}]=[0.3,2]$ (right upper panel), $[\underline{b}, \bar{b}]=[1,5]$ (left lower panel), $[\underline{b}, \bar{b}]=[1,10]$ (right lower panel). 


\section{Appendix: proofs}

We begin with an auxiliary result, which was used in Section 3 and is also required for several steps in the proofs of our main results.

Lemma A.1. For any fixed $T>0$ and $b>0$ the functions

$$
f_{1}(x, b)=\frac{x}{(x+b)^{2}}, \quad f_{2}(x, b)=\frac{x^{2}}{(x+b)^{2}}, \quad f_{3}(x, b)=\frac{x \log x}{(x+b)^{2}}
$$

form a Chebyshev system on the interval $(0, T]$.

Proof of Lemma A1. It follows from Karlin and Studden (1966) that the functions $g_{1}(x), \ldots, g_{m}(x)$ generate a Chebyshev system on the interval $(c, d]$ if and only if for any $\alpha_{1}, \ldots, \alpha_{m}$ not all equal to zero the function

$$
\sum_{i=1}^{m} \alpha_{i} g_{i}(x)
$$

vanishes in the interval $(c, d]$ no more than $m-1$ times. To prove the Lemma, we suppose on the contrary that there exists a function

$$
g(x)=\frac{\alpha_{1} x}{(x+b)^{2}}+\frac{\alpha_{2} x^{2}}{(x+b)^{2}}+\frac{\alpha_{3} x \ln x}{(x+b)^{2}}, \sum_{i=1}^{3} \alpha_{i}^{2} \neq 0,
$$

which vanishes 3 or more times on the interval $(0, T]$. If we let $v(x)=x^{2} /(x+b)^{2}, \psi_{1}(x)=1 / x$, $\psi_{2}(x)=1, \psi_{3}(x)=(\ln x) / x$, the supposition is the same as asserting the function

$$
g(x)=v(x)\left[\alpha_{1} \psi_{1}(x)+\alpha_{2} \psi_{2}(x)+\alpha_{3} \psi_{3}(x)\right]
$$

vanishes 3 or more times on the interval $(0, T]$. Let $0<x_{1}<x_{2}<x_{3} \leq T$ denote the three distinct points with $g\left(x_{i}\right)=0(i=1,2,3)$ and note that $g(x) \rightarrow 0$ as $x \rightarrow 0$. It follows from here that there exists three points $\tilde{x}_{1} \in\left(0, x_{1}\right), \tilde{x}_{2} \in\left(x_{1}, x_{2}\right)$ and $\tilde{x}_{3} \in\left(x_{2}, x_{3}\right)$ such that $g^{\prime}\left(\tilde{x}_{i}\right)=0$, $i=1,2,3$. It is easy to check that $v(x)>0, v^{\prime}(x)>0$ whenever $x \in(0, T]$. Since

$$
g^{\prime}(x)=v^{\prime}(x) \sum_{i=1}^{3} \alpha_{i} \psi_{i}(x)+v(x) \sum_{i=1}^{3} \alpha_{i} \psi_{i}^{\prime}(x),
$$

we have

$$
\operatorname{sign} \sum_{i=1}^{3} \alpha_{i} \psi_{i}^{\prime}\left(\tilde{x}_{j}\right)=(-1)^{j} \varepsilon, j=1,2,3,
$$

where $\varepsilon$ is either 1 or -1 . Consequently, there exists two points $\bar{x}_{1}, \bar{x}_{2}$ with $\bar{x}_{1} \in\left(\tilde{x}_{1}, \tilde{x}_{2}\right)$, $\bar{x}_{2} \in\left(\tilde{x}_{2}, \tilde{x}_{3}\right)$ such that

$$
\bar{g}(x):=\sum \alpha_{i} \psi_{i}^{\prime}\left(\bar{x}_{j}\right)=0 j=1,2 .
$$

However,

$$
\begin{aligned}
\bar{g}(x) & =-\frac{\alpha_{1}}{x^{2}}-\frac{\alpha_{3} \ln x}{x^{2}}+\frac{\alpha_{3}}{x^{2}}= \\
& =\frac{1}{x^{2}}\left(\beta_{1}-\alpha_{3} \ln x\right), \beta_{1}=\alpha_{3}-\alpha_{1} .
\end{aligned}
$$


Due to the monotonicity of the logarithm the function $\bar{g}(x)$ can vanish at two distinct points $0<\bar{x}_{1}<\bar{x}_{2}$ if and only if $\alpha_{3}=\alpha_{1}=0$. But in this case we had $g(x)=\alpha_{2} x^{2} /(x+b)^{2}, \alpha_{2} \neq 0$ and, therefore, $g(x) \neq 0$ whenever $x \in(0, T]$. The obtained contradiction proves that the functions $f_{1}$, $f_{2}, f_{3}$ generate a Chebyshev system on $(0, T]$.

\subsection{Proof of Theorem 2.1}

Recalling the definition of the vector $f(x, a, b, h)$ in $(1.2)$ and the definition $\tilde{\xi}(\{x\})=\xi\left(\left\{x^{1 / h}\right\}\right)$, it follows from a straightforward calculation that

$$
f\left(x^{1 / h}, a, b, h\right)=S \cdot f(x, a, b, 1),
$$

where the matrix $S$ is defined by

$$
S=\left(\begin{array}{ccc}
1 & 0 & 0 \\
0 & 1 & 0 \\
0 & 0 & 1 / h
\end{array}\right)
$$

If $\xi$ puts mass $w_{i}$ at $x_{i}$, then the design $\tilde{\xi}$ puts mass $w_{i}$ at $x_{i}^{h}$, which implies

$$
\begin{aligned}
M(\tilde{\xi}, a, b, 1) & =\sum_{i=1}^{n} w_{i} f\left(x_{i}^{h}, a, b, 1\right) f^{T}\left(x_{i}^{h}, a, b, 1\right) \\
& =\sum_{i=1}^{n} w_{i} S^{-1} f(x, a, b, h) f^{T}(x, a, b, h) S^{-1} \\
& =S^{-1} M(\xi, a, b, h) S^{-1}
\end{aligned}
$$

and the assertion of Theorem 2.1 follows because the $D$ - and $D_{1}$-criterion are based on the determinants of the information matrices $M(\xi, a, b, h)$ and $\tilde{M}(\xi, a, b, h)$ (recall that $\tilde{M}$ is obtained from $M$ by deleting the last row and column).

\subsection{Proof of Theorem 3.1}

From the definition of the vector $f(x, b)=f(x, 1, b, 1)$ in $(2.3)$, the matrix $M(\xi, b)=M\left(\xi_{D}, 1, b, 1\right)$ in (2.2) and the equivalence theorem of Kiefer and Wolfowitz (1960), it follows that a design $\xi_{b}$ is locally $D$-optimal on the interval $[0,1]$ if and only if the inequality

$$
d\left(x, \xi_{b}^{D}\right)=f^{T}(x, b) M^{-1}\left(\xi_{b}^{D}, b\right) f(x, b) \leq 3
$$

holds for all $x \in[0,1]$ with equality at the support points of $\xi_{b}^{D}$. From the equation $f(0, b)=0$ it follows that 0 is not a support point of the locally $D$-optimal design. Let $0<x_{1}^{*}<\ldots<x_{n}^{*} \leq 1$ be the support points of the locally $D$-optimal design

$$
\xi_{b}^{D}=\left(\begin{array}{ccc}
x_{1}^{*} & \ldots & x_{n}^{*} \\
w_{1}^{*} & \ldots & w_{n}^{*}
\end{array}\right)
$$


and let $g(x)=(x+b)^{4}\left\{d\left(x, \xi_{b}^{D}\right)-3\right\}$. We observe that (5.1) is equivalent to the inequality $g(x) \leq 0$ with equality at the support points and so

$$
\begin{aligned}
g\left(x_{i}^{*}\right) & =0, \quad i=1, \ldots, n \\
g^{\prime}\left(x_{i}^{*}\right)=0, & i=1, \ldots, n-1 \\
g^{\prime}\left(x_{n}^{*}\right)=0, & \text { if } x_{n}^{*}<1 .
\end{aligned}
$$

Note that $n \geq 3$ because otherwise $M\left(\xi_{b}^{D}, b\right)$ is singular. A simple calculation shows that

$$
\psi(x):=\left(x^{2} g^{(4)}(x)\right)^{\prime}=\alpha_{0}+\alpha_{1} x+\frac{\alpha_{2}}{x}
$$

for some constants $\alpha_{0}<0, \alpha_{1}>0, \alpha_{2} \in \mathbb{R}$ which shows that the function $x \psi(x)$ has at most two roots in the interval $[0,1]$. Consequently, the function $g(x)$ has at most 7 roots on the interval $[0,1]$ counted with their multiplicities. A careful counting of the multiplicities yields $n=3$. If $\alpha_{2}>0$ the same argument shows for the largest support point $x_{3}^{*}=1$. On the other hand, if $\alpha_{2} \leq 0$, the function $x \psi(x)$ has one root in the interval $[0, \infty)$ and the proof of the property $x_{3}^{*}=1$ follows by similar arguments as given in Dette, Melas, Pepelyshev and Strigul (2003). A standard argument [see Silvey (1980), Lemma 5.1.3] shows that $\xi_{b}^{D}$ must have equal weights at its support points.

Secondly, if there would exist two $D$-optimal designs, say $\xi_{b}^{(1)}$ and $\xi_{b}^{(2)}$, then these arguments show that $\xi_{b}^{(1)}$ and $\xi_{b}^{(2)}$ have equal weights at three support points. Consequently, the log-concavity of the determinant criterion implies that the design $\frac{1}{2}\left(\xi_{b}^{(1)}+\xi_{b}^{(2)}\right)$ is also $D$-optimal with at least four support points. This contradicts the fact that any locally $D$-optimal design has three support points. Finally, the existence of the $D$-optimal design on the interval $[0,1]$ follows from the continuity of the vector $f(x, b)$ and the compactness of the design space.

In the general case, where the design space is given by the interval $[0, T]$ we consider for each design $\xi$ on the interval $[0,1]$ the design $\xi_{T}$ on $[0, T]$ induced by the transfomation $x \rightarrow T x$, that is $\xi_{T}(\{x\})=\xi\left(\left\{\frac{x}{T}\right\}\right)$. In this case we have by a simple calculation

$$
\left|M\left(\xi_{T}, T b\right)\right|=c \cdot|M(\xi, b)|,
$$

where the constant $c$ does not depend on the design. Consequently, the locally $D$-optimal design on the interval $[0, T]$ is obtained from the local $D$-optimal design $\xi_{b / T}^{D}$ on the interval $[0,1]$ by multiplying the support points with the parameter $T$.

\subsection{Proof of Theorem 3.2}

We first consider the design problem on the interval $[0,1]$. Let $0<x_{1}^{*}<x_{2}^{*}<x_{3}^{*} \leq 1$ denote the corresponding Chebyshev points and recall the definition of the weights $w_{i}^{*}$ in (3.9) and (3.11). With the notation

$$
\bar{F}=\left(f_{i}\left(x_{j}^{*}, b\right)\right)_{i, j=1}^{3}
$$

it follows from Dette, Melas and Pepelyshev (2003) that the design $\xi^{D_{1}}$ with weights $w_{i}^{*}$ at the points $x_{i}^{*}$ is locally $D_{1}$-optimal (in other words $e_{3}$-optimal) for the EMAX model (1.2) with respect 
to the parameter $(b, 1)$ if and only if $\bar{F} w^{*}=e_{3}=(0,0,1)^{T}$ and $\alpha_{3} \neq 0$, where $\alpha_{3}$ is the coefficient of $x \log x /(x+b)^{2}$ in the Chebyshev polynomial $(3.5)$. Here $w^{*}=\left(w_{1}^{*}, w_{2}^{*}, w_{3}^{*}\right)$ is the vector of weights of the design $\xi^{D_{1}}$. A direct calculation shows that the equation $\bar{F} w^{*}=e_{3}$ is satisfied. Assume that $\alpha_{3}=0$, then it follows from (3.5) and (3.6) that

$$
\psi\left(x_{j}^{*}\right)=\frac{x_{j}^{*}}{\left(b+x_{j}^{*}\right)^{2}}\left\{\alpha_{1}+\alpha_{2} x_{j}^{*}\right\}=(-1)^{j}, \quad j=1,2,3,
$$

which is impossible, because the function $\psi$ has only one root, if $\alpha_{3}=0$. This shows that the design with weights $w_{1}^{*}, w_{2}^{*}$ and $w_{3}^{*}$ at the Chebyshev points $x_{1}^{*}, x_{2}^{*}$ and $x_{3}^{*}$ is in fact locally $D_{1}$-optimal for the EMAX model (for $h=1$ ). We now prove that $x_{3}^{*}=1$. For this we assume the contrary, i.e. $0<x_{1}^{*}<x_{2}^{*}<x_{3}^{*}$ and note that the Chebyshev polynomial (3.5) can be written as

$$
\psi(x)=z(x) \sum_{i=1}^{3} \alpha_{i} \bar{f}_{i}(x)
$$

where $z(x)=x^{2} /(x+b)^{2}, \bar{f}_{1}(x)=1, \bar{f}_{2}(x)=1 / x, \bar{f}_{3}(x)=(\log x) / x$. We have $\psi\left(x_{i}^{*}\right)=(-1)^{i}, \psi^{\prime}\left(x_{i}^{*}\right)=$ $0(i=1,2,3), z(x)>0, z^{\prime}(x)>0$ for $x \in(0, \infty)$. Consequently, the function

$$
\tilde{\psi}(x)=\frac{\partial}{\partial x} \sum_{i=1}^{3} \alpha_{i} \bar{f}_{i}(x)=\left(\alpha_{3}-\alpha_{2}\right) \frac{1}{x^{2}}-\alpha_{3} \frac{\log x}{x^{2}}
$$

is positive at $x_{1}^{*}$ and $x_{3}^{*}$ and negative at $x_{2}^{*}$, which means that $\tilde{\psi}(x)$ vanishes at (at least) two points $t_{1} \in\left(x_{1}^{*}, x_{2}^{*}\right)$ and $t_{2} \in\left(x_{2}^{*}, x_{3}^{*}\right)$. However,

$$
x^{2} \tilde{\psi}(x)=\left(\alpha_{3}-\alpha_{2}\right)-\alpha_{3} \log x
$$

vanishes at at most one point contradicting to the assumption $x_{3}^{*}<1$.

For the proof of the proof of the corresponding statement on the interval $[0, T]$ we define

$$
G\left(x_{1}, x_{2}, x_{3}, b\right)=\max _{w}\left(e_{3}^{T} M^{-1}(\xi, b) e_{3}\right)^{-1}
$$

(in other words: for fixed support points, say $x_{1}, x_{2}, x_{3}$, we determine the weights of the $D_{1}$-optimal design). It follows from Pukelsheim and Torsney (1993) and a straightforward calculation that

$$
G\left(x_{1}, x_{2}, x_{3}, b\right)=\left(\frac{x_{3} x_{2}\left(x_{3}-x_{2}\right)\left(b+x_{1}\right)^{2}+x_{1} x_{3}\left(x_{3}-x_{1}\right)\left(b+x_{2}\right)+x_{1} x_{2}\left(x_{2}-x_{1}\right)\left(b+x_{3}\right)}{b x_{1} x_{2} x_{3}\left[\left(x_{3}-x_{2}\right) \log x_{1}-\left(x_{3}-x_{1}\right) \log x_{2}+\left(x_{2}-x_{1}\right) \log x_{1}\right.}\right.
$$

and it is easy to see that for any $T \neq 0$,

$$
G\left(T x_{1}, T x_{2}, T x_{3}, T b\right)=G\left(x_{1}, x_{2}, x_{3}, b\right) .
$$

The assertion regarding the $D_{1}$-optimal design on the interval $[0, T]$ is now obvious. 


\subsection{Proof of Lemma 4.1}

Let $\Xi_{T}$ denote the class of all designs on the interval $[0, T]$. If $\xi_{1} \in \Xi_{1}$ and $\xi_{T} \in \Xi_{T}$ is related to $\xi_{1}$ by the transformation $\xi_{T}(\{x\})=\xi\left(\left\{\frac{x}{T}\right\}\right)$, then it follows from (5.3)

$$
\begin{aligned}
\min _{b \in[b, \bar{b}]} \frac{\left|M\left(\xi_{T}, b\right)\right|}{\max _{\eta_{T} \in \Xi_{T}}\left|M\left(\eta_{T}, b\right)\right|} & =\min _{b \in[\underline{b}, \bar{b}] \max _{\eta_{1} \in \Xi_{1}} \frac{\left|M\left(\xi_{1}, \frac{b}{T}\right)\right|}{\left.\max _{1}, \frac{b}{T}\right) \mid}} \\
& =\min _{b \in[\underline{b} / T, \bar{b} / T]} \frac{\left|M\left(\xi_{1}, b\right)\right|}{\max _{\eta_{1} \in \Xi_{1}}\left|M\left(\eta_{1}, b\right)\right|}
\end{aligned}
$$

which proves the assertion. The justification for the corresponding assertion for the $D_{1}$-optimality criterion of the Lemma is similar and is omitted.

\section{References}

R.J.H. Beverton and S.J. Holt (1957). On the Dynamics of Exploited Fish Populations. London: Her Majesty's Stationary Office.

M. Bezeau and L. Endrenyi (1986). Design of experiments for the precise estimation of doseresponse parameters: the Hill equation. J. Theor. Biol. 123, 415-430.

E.P.J. Boer, D. Rasch and E.M.T. Hendrix (2000). Local optimal designs in non-linear regression: A case study for the Michaelis-Menten function. Balakrishnan, N. (ed.) et al., Advances in Stochastic Simulation Methods, Selected papers at the 3rd St. Petersburg workshop on simulation, St. Petersburg, Russia, June 28 - July 3, 1998. Boston: Birkhäuser. Statistics for Industry and Technology, 177-188.

H. Chernoff (1953). Locally optimal designs for estimating parameters. Ann. Math. Statist. 24, 586-602.

A. Cornish-Browden (1995). Fundamentals of Enzyme Kinetics (Rev. Ed.). Portland Press, London.

N.A.C. Cressie and D.D. Keightley (1979). The underlying structure of a direct linear plot with applications to the analysis of hormone-receptor interactions. Journal of Steroid Biochemistry 11, 1173-1180.

H. Dette (1997). Designing experiments with respect to standardized optimality criteria. J. Roy. Statist. Soc., Ser. B, 59, No.1, 97-110.

H. Dette and S. Biedermann (2003). Robust and efficient designs for the Michaelis-Menten model. J. Amer. Stat. Assoc., 98, 679-686.

H. Dette, V.B. Melas, A. Pepelyshev and N. Strigul (2003). Efficient design of experiment in the Monod model. J. Roy. Statist. Soc., Ser. B, 65, 725-742.

H. Dette and W.K. Wong (1999). E-optimal designs for the Michaelis-Menten model. Statistics \& Probability Letters 44, No.4, 405-408. 
R.G. Duggleby (1979). Experimental designs for estimating the kinetic parameters for enzymecatalysed reactions. J. Theor. Biol. 81, 671-684.

G. Dunn (1988). Optimal designs for drug, neurotransmitter and hormone receptor assays. Statistics in Medicine 7, 805-815.

A. C. Greenwood, E. M. Landaw and T. H. Brown. (1999). Testing the fit of a quantal model of neurotransmission. Biophysical Journal, 76, 1847-1855.

W.W. Hay, H.K. Meznarich, J.E. DiGiacomo, K. Hirst and G. Zerbe (1988). Effects of insulin and glucose concentration on glucose utilization in fetal sheep. Pediatric Research 23, 381-387.

N.H.G. Holford and L.B. Sheiner (1981). Understanding the dose-effect relationship: clinical application of pharmacokinetic - pharmacodynamic models. Clin. Pharmacokinet. 6, 429-453.

L.A. Imhof (2001). Maximin designs for exponential growth models and heteroscedastic polynomial models. Ann. Statist. 29, No.2, 561-576.

L.A. Imhof and W.K. Wong (2000). A graphical method for finding maximin designs. Biometrics $56,113-117$.

R.J. Jennrich (1969). Asymptotic properties of non-linear least squares estimators. Ann. Math. Statist. 40, 633-643.

S. Johansen (1984). Functional Relations, Random Coefficients and Nonlinear Regression, with Application to Kinetic Data. Lecture Notes in Statistics, 22. Springer, N.Y.

J. Kiefer and J. Wolfowitz (1960). The equivalence of two extremum problems. Can. J. Math. $12,363-366$.

J. Lopez-Fidalgo and W.K. Wong (2002). Optimal designs for the Michaelis-Menten model. J. Theor. Biol. 215, No.1, 1-11.

P. J. Lupinacci and D. Raghavarao (2000). Designs for testing lack of fit for a nonlinear doseresponse curve model. Journal of Biopharmaceutical Statistics, 10, 45-53.

P. J. Lupinacci and D. Raghavarao (2003). D-optimal designs for the Michaelis-Menten model under various error variance structures. Dept. of Mathematical Sciences Technical Report. Villanova University, Villanova, PA.

J.N.S. Matthews and G. C. Allcock (2004) Optimal designs for Michalelis-Menten Kinetic studies. Statistics in Medicine, 23, 477-491.

P.J. Meftin, R.A. Winkle, T.F. Blaschke, J. Fitzgerald and D.C. Harrison (1977). Clin. Pharmacol. Ther. 22, 42-

C.H. Müller (1995). Maximin efficient designs for estimating nonlinear aspects in linear models. J. Statist. Plann. Inference 44, No.1, 117-132.

F. Pukelsheim (1993). Optimal design of experiments. Wiley, N.Y.

D. Rasch (1990). Optimum experimental design in nonlinear regression. Commun. Statist., Theor. Meth. 19, 4789-4806.

S.D. Silvey (1980). Optimal design. Chapman and Hall, London. 
D. Song and W.K. Wong (1998). Optimal two point designs for the Michaelis-Menten model with heteroscedastic errors. Commun. Statist., Theory Math. 27, No.6, 1503-1516.

S.M. Stigler (1971). Optimal experimental design for polynomial regression. J. Amer. Stat. Assoc., 66, 311-318.

W.J. Studden (1982). Some robust type $D$-optimal designs in polynoimal regression. J. Amer. Stat. Assoc., 77, 916-921. 